\title{
Artificial Intelligence-Based Polyp Detection in Colonoscopy: Where Have We Been, Where Do We Stand, and Where Are We Headed?
}

\author{
Thomas Wittenberg $^{\mathrm{a}} \quad$ Martin Raithel $^{\mathrm{b}}$ \\ ${ }^{a}$ Fraunhofer Institute for Integrated Circuits IIS, Erlangen, Germany; ${ }^{\text {b } M a l t e s e r ~ W a l d k r a n k e n h a u s ~ S t . ~ M a r i e n, ~}$ \\ Erlangen, Germany
}

\section{Keywords}

Artificial intelligence $\cdot \mathrm{Al} \cdot$ Colonoscopy $\cdot$ Adenoma and polyp detection $\cdot$ History

\begin{abstract}
Background: In the past, image-based computer-assisted diagnosis and detection systems have been driven mainly from the field of radiology, and more specifically mammography. Nevertheless, with the availability of large image data collections (known as the "Big Data" phenomenon) in correlation with developments from the domain of artificial intelligence (Al) and particularly so-called deep convolutional neural networks, computer-assisted detection of adenomas and polyps in real-time during screening colonoscopy has become feasible. Summary: With respect to these developments, the scope of this contribution is to provide a brief overview about the evolution of Al-based detection of adenomas and polyps during colonoscopy of the past 35 years, starting with the age of "handcrafted geometrical features" together with simple classification schemes, over the development and use of "texture-based features" and machine learning approaches, and ending with current developments in the field of deep learning using convolutional neural networks. In parallel, the need and necessity of large-scale clinical data will be discussed in order to develop such methods, up to commercially available Al products for automated detection of polyps (adenoma and benign neoplastic lesions). Finally, a short view into the future is made regarding further possibilities of Al methods within colonoscopy. Key Messages: Research of image-based lesion detection in colonoscopy data has a 35-year-old history. Milestones such
\end{abstract}

as the Paris nomenclature, texture features, big data, and deep learning were essential for the development and availability of commercial Al-based systems for polyp detection.

๑) 2020 S. Karger AG, Basel

\section{Introduction}

Since the beginning of the interdisciplinary research field "medical image analysis," there have been many different applications and challenges to be solved within the domain of "computer-assisted detection," commonly abbreviated as CADe. In contrast to "computer-assisted diagnosis" (CADx), where the diagnostic differentiation of an already located and segmented image region or volume into various classes is supported by a computer, CADe is concerned with the preceding step of the detection and location (and sometimes the delineation) of conspicuous regions or volumes within medical images, which could be related to a potential lesion.

Originally, CADe approaches were applied to the detection of micro-calcification and mass lesions in digitized or digital mammographies, as (according to the WHO) breast cancer is the most common form of cancer within Europe. As the mortality of breast cancer increased in the late 1980s, screening programs for breast cancer were established throughout Europe as early prevention measures. Simultaneously, from the technical side, a strong focus was put on the investigation and development of image-based CADe methods and systems for mammography, in order to support the radiologist with an "electronic second reader." 
Recently, novel methodical approaches and breakthroughs from the field of "artificial intelligence" (AI) and specifically from "deep learning" have improved the automated image-based breast-cancer detection [1-3] and have pushed their possibilities to a new diagnostic level. "Deep Learning," being a novel research field in the domain of AI, is based on the training and application of so-called deep convolutional neural networks (DCNNs), a computer simulation of networks consisting of some hundreds of thousands of artificial neurons, whose interconnections and weights are optimized with respect to certain discriminative tasks, such as lesion detection. One booster of these developments was the collection and public availability of digital and digitized mammographic reference data sets such as the DDSM database [4]. This data collection consists of over 2,600 cases and includes malignant, benign, and normal cases along with annotated lesions and verified pathology information.

Even though the native digitalization of endoscopy and colonoscopy image data (meaning their direct digital availability from the endoscopy systems and without the need for a time-consuming post-conversion from analogue VHS or UMATIC cartridges to digital video data) has been around for approximately 15 years, the related developments regarding the possibilities of automated detection, localization, delineation, and classification of polyps (and other malignant lesions) in gastroscopy and colonoscopy videos have a similar time delay with respect to CADe in mammography.

\section{Scope of This Contribution}

The scope of this work is to provide a brief commentary on the evolution of AI-based detection of polyps (benign and adenomatous lesions, for example colloquial polyps) as the predecessor of colorectal carcinoma, during colonoscopy of the past 35 years. This overview starts with the age of handcrafted geometrical features together with simple decision schemes, covers the development and use of texture-based features ("texture" meaning here the visual surface characteristics and appearance of the colon tissue) and machine learning approaches, and ends with current developments in the field of deep learning using convolutional neural networks. In parallel, the need and necessity of clinical data will be discussed in order to develop such methods, up to commercially available AI-based products for automated polyp detection. The term "polyp" will be used throughout this article to describe adenoma and benign neoplastic lesions of the colon, although histologically various polyp etiologies exist. Finally, a view into the future is made to consider further possibilities of AI-based methods within colonoscopy.

\section{Three Generations of Automated Polyp Detection}

According to William Vorhies [5] (who in turn cites John Launchbury from DARPA), there have been three generations (or "ages" or "waves") of "artificial intelligence", a research field from informatics which has been around for over 60 years and which is currently newly connotated with the aforementioned "deep learning" approaches, mainly using DCNNs. Thus, in order to better understand the current achievements of "deep learning" with respect to automated polyp detection for screening colonoscopy, let us have a brief look at the abovementioned three ages of AI and their influence on polyp detection.

According to Vorhies [5], the history and future of AI can coarsely be divided into the ages or waves of:

1. handcrafted knowledge,

2. statistical learning, and

3. contextual adaptation (Fig. 1).

As "waves" (or "ages") tend to come one after the other, they also interact with and build on top of each other, meaning that ideas, experiences, and results of earlier waves (or ages) are not over, but are still around and strongly influence successive developments. Explicitly, older technologies and results from earlier AI developments are still useful and are in fact still used [5]. Even though Vorhies' [5] division of AI ages represents quite a high level, it provides a good idea of how to structure the developments of the past with respect to automated polyp detection.

\section{Age of Handcrafted Knowledge}

The "first wave" of polyp detection approaches can be characterized by "knowledge-based" methods, where socalled handcrafted features related to human observations (and anatomical knowledge) were implemented and evaluated on some small sets (of some dozen) colonoscopic (mostly black and white) images, which have retrospectively been digitized. Usually in the first step a combination of image-to-image pre-processing operation is performed, such as color conversion, linear or nonlinear image smoothing and noise reduction, aperture detection and segmentation [6], or edge localization, as depicted in Figures 2 and 3. Under the assumption that the color or hue of an image region provides an indication of a potential lesion, such pre-processing steps also include the transformation of the endoscopic images into other color spaces, such as CieLAB or HSV [7-10]. Then, in a second step, handcrafted features are extracted, such as the description of regions of similar color appearance [6-9], or the description of the haustra edges [11, 12], under the assumption that possible polyps will distort their curvatures $[13,14]$, as depicted in Figure 2d. Also, the fit- 


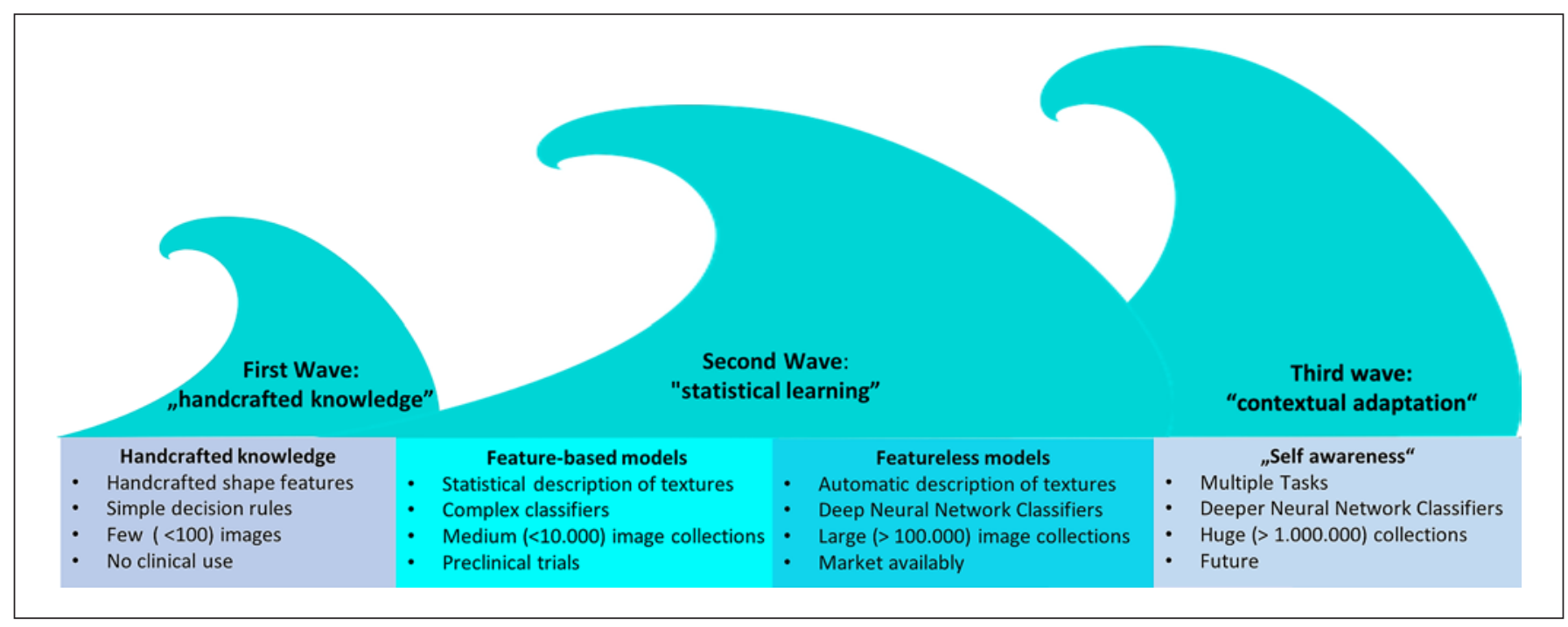

Fig. 1. Three waves ("generations" or "ages”) of AI with respect to the progression of automated polyp detection.
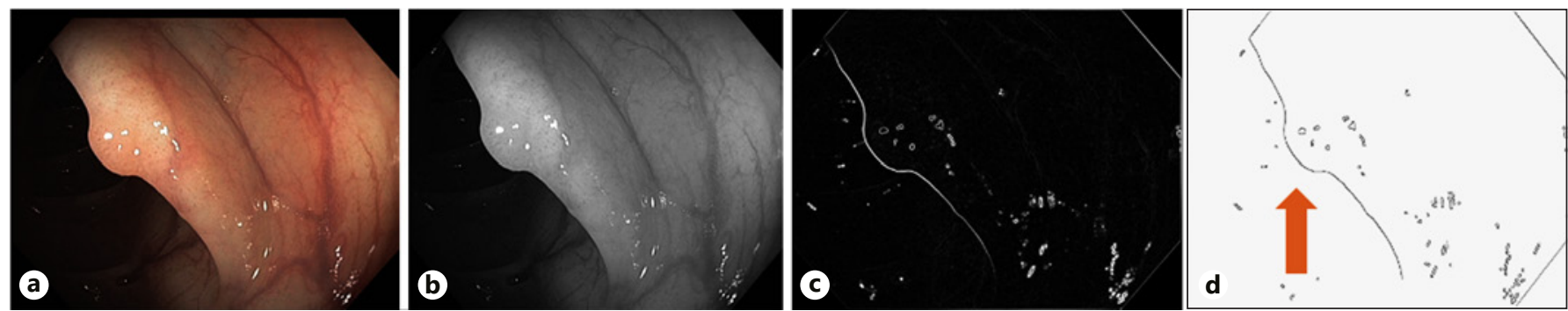

Fig. 2. Examples of early polyp detection based on image processing and handcrafted features: original colonoscopy image (a), color conversion to green channel (b), edge detection using the Canny operator (c), detection of convex curvatures as an indicator of possible polyps (d).
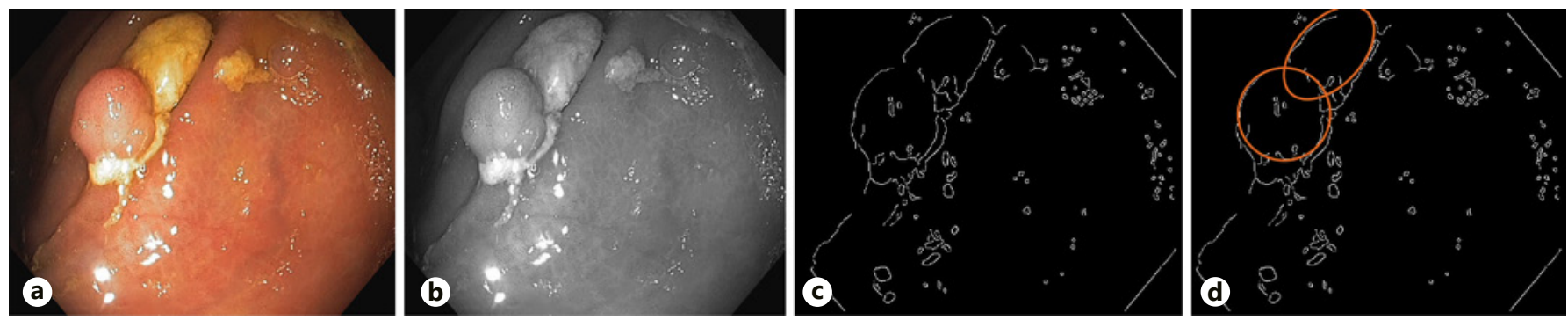

Fig. 3. Example of early polyp detection based on image processing and shape detection: original colonoscopy image (a), color conversion to gray-level information (b), edge detection using the Canny edge detector (c), polyp detection using ellipse fitting (d).

ting of elliptical shapes into the edge image [15] was proposed and hereby considering strong edges correlated with the ellipses, shown in Figure 3d. In a third step, the extracted geometrical features were used to decide if a polyp is present or not, usually using some simple decision rules based on a set of local thresholds. A brief overview of approaches for polyp detection based on handcrafted knowledge is provided by Ameling et al. [16].
These approaches have only been academic, as the number of images available for algorithm development and evaluation was quite sparse and in the range of some dozen digitized colonoscopy images. Furthermore, the achieved results were not really useful in a clinical sense as they only considered large prominent polyps and neglected the small and flat ones. 


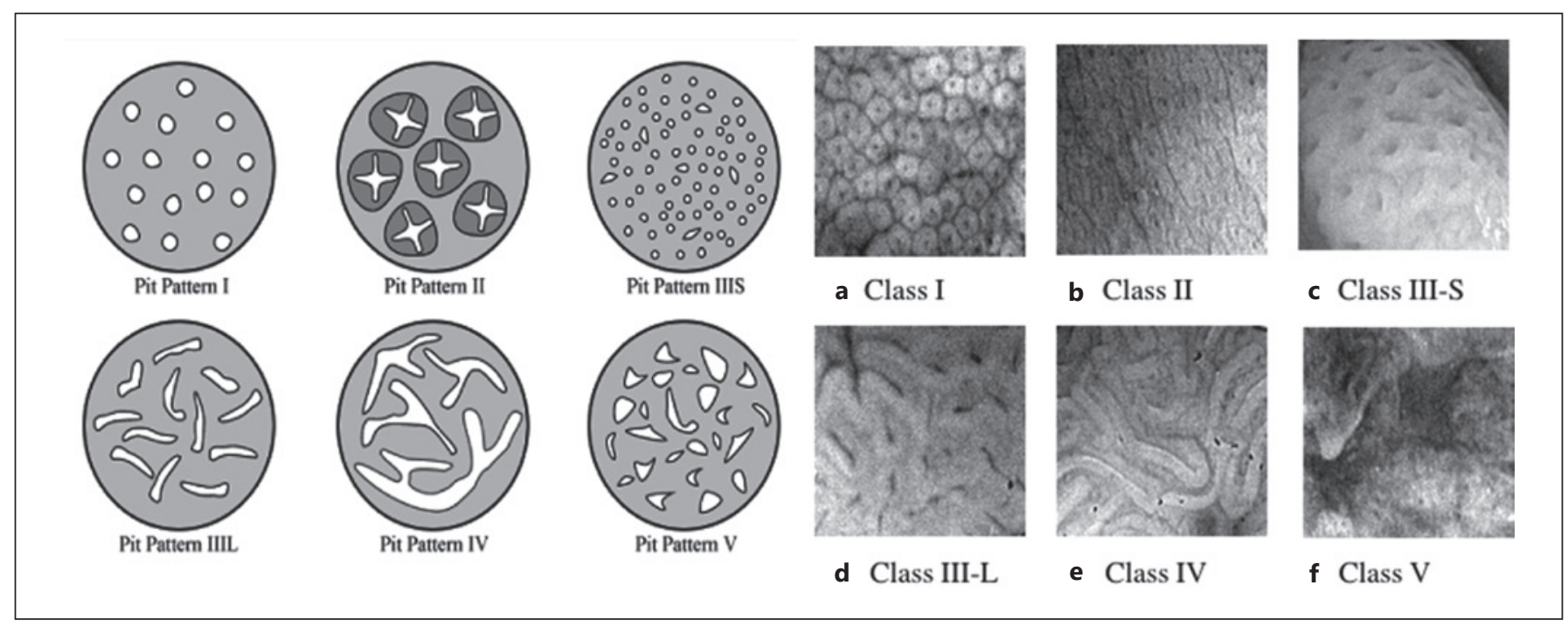

Fig. 4. Visual examples of the pit patterns according to Kudo et al. [17] and zoom-endoscopic examples for each pattern type (a-f), as used by Häfner et al. [37].

\section{The Age of Statistical Learning}

The second age of AI, denoted as the age of "statistical leaning," first mentioned around the 1990s, can conceptually be split up into halves, namely the eras of "featurebased" and the era of "featureless" modelling [5].

\section{Era of Feature-Based Modelling}

With this first era of "feature-based" modelling three different developments can be observed with respect to automated image-based polyp detection.

Medical View: Pit Patterns and Paris Nomenclature

On the medical side, one milestone was - after the establishment of high-resolution endoscopy sensors - the definition and description of the "pit pattern" of a given adenomatous lesion in 1998 by Kudo et al. [17]. Later on, the different forms of neoplastic (adenomatous) growth within the gastrointestinal tract were categorized in the wellknown "Paris classification" for early or superficial neoplastic lesions in the esophagus, stomach, and colon [18]. With these two standardized classification tools - (i) "pit pattern" at the surface of a lesion and (ii) description of the type of neoplastic growth (e.g., polypoid, flat and superficial, or excavated shape or ulcerated, respectively) - for the first time a structured nomenclature was available to visually describe and standardize nomenclature, encompassing the different types, appearances, and stages of polyps in the colon (Fig. 4). Even though in the clinical context "pit patterns" are used for the differentiation of a given lesion to predict or estimate visually the final histological result and to stratify endoscopic treatment, from the technical side, the underlaying textures were further used for lesion detection (CADe).
Technical View: Statistical Description of Textures

Secondly, in the field of image processing or image analysis, the idea of a machine-based description of "texture" or "structure" materialized. Here, the goal was to describe and characterize the (manually or automatically delineated) visual surface characteristics and appearance of a patch or region in an endoscopic image by a set of complex mathematical "texture" or "structure" features.

Over the past 30 years, many texture descriptors have been developed. For example, Humeau-Heurtier [19] recently categorized these descriptors into various different classes, including statistical, structural, transform-based, model-based, graph-based, learning-based, and entropybased approaches. Statistical approaches for texture description are based on the gray-level representations of images or image regions and the spatial distribution of each gray value. Structural approaches are based on the assumption that each texture can be represented as a repetition of basic textual elements, also referred to as primitives. Transform-based approaches convert the image into another coordinate system (e.g., frequency space) where textual differences become more significant. Model-based approaches, on the other hand, try to fit a mathematical model (e.g., network structures or random fields) to the given input, whereas graph-based approaches rely on graphs constructed from the input data.

The application of such texture descriptors or texture features for the objective of automated lesion and polyp detection in colonoscopy images was also motivated by the abovementioned description of pit patterns, as these (as well as the healthy tissue surrounding them) visually denote different types of structures and textures which can be described by such features (Fig. 4). 


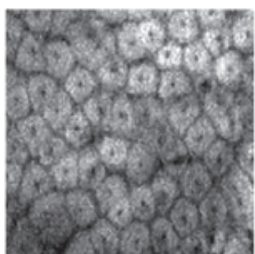

a Class I

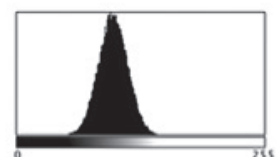

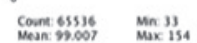
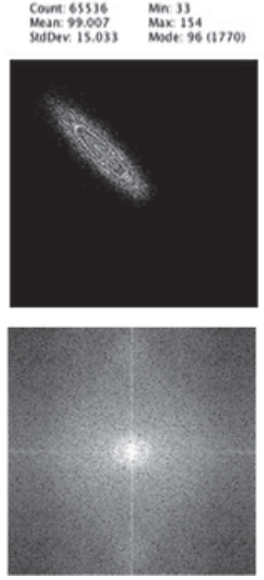

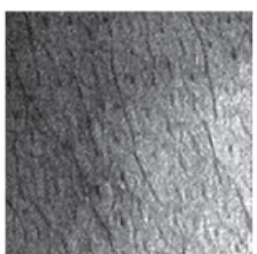

b Class II
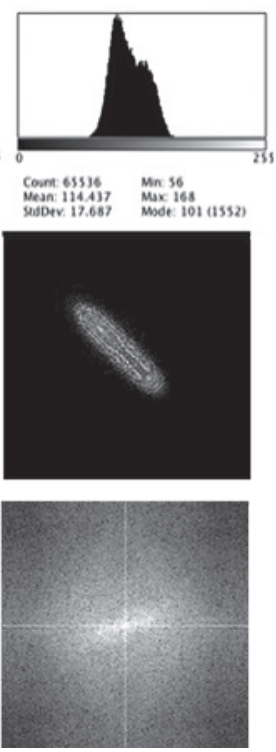

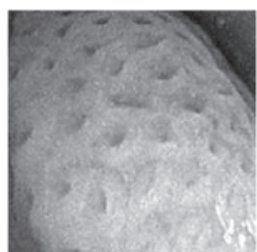

c Class III-S
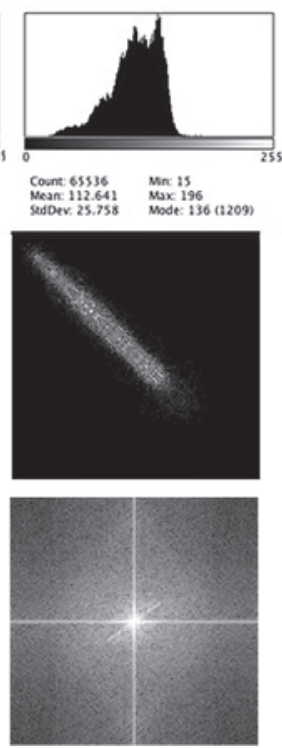

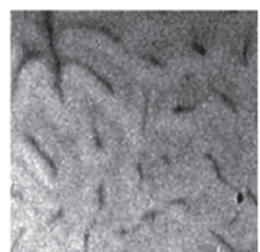

d Class III-L
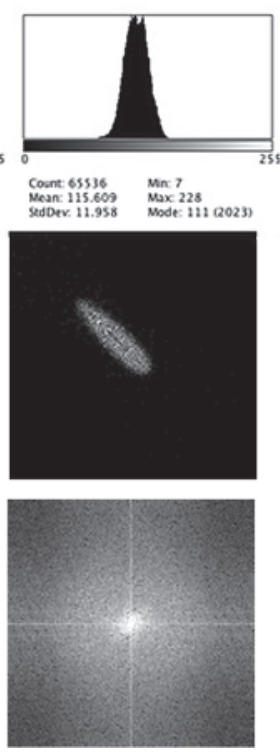

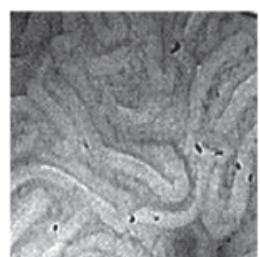

e Class IV
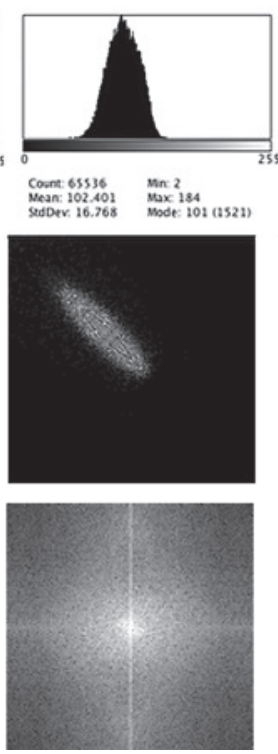

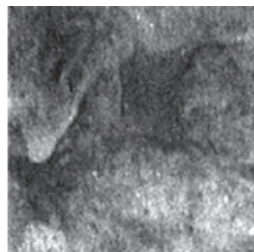

f Class V
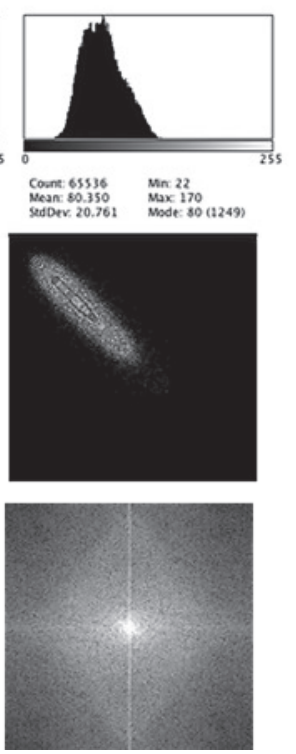

Fig. 5. a-f Zoom-endoscopic examples for each pit pattern type (first row) and depiction of different meta-representations used for texture description. Second row, gray-scale histograms; third row, gray-level co-occurrence matrices; fourth row, Fourier-transformed images. Original images from Häfner et al. [37].

Typical examples for the statistical approach suggested and applied for the detection and description of suspicious lesions in the colon are features computed from gray-level and color histograms $[9,20,21]$ (Fig. 5, second row), from gray-level co-occurrence matrices, (Fig. 5, row three) [22-29], sum-and different histograms [20, 21], texture spectrum [10], or local binary patterns $[20,21$, 30-32]. Examples for structural approaches were statistical-geometrical features [20] or features obtained from fractal analysis [29]. Features obtained from the Fourier transform [33-35] (Fig. 5, bottom row) or the Wavelet transform $[10,25,31,36,37]$ are examples for the transform-based approaches, where the colonoscopy images are transformed into another visual representation from where descriptive features are extracted. Finally, Eigen textures [38] are an example for the model-based approach. As already mentioned in the previous section, the color of a suspicious lesion and its visible difference to the surrounding tissue (the color gradient) are also known to provide important indications for the detection of polyps, Thus, also color-extended texture features have been proposed $[20,21,37,39,40]$. Finally, under the assumption that a polyp to be detected usually appears in more than one consecutive image frame, also the temporal progression of a lesion over time has been considered as a feature [38].

\section{Machine Learning for Polyp Detection}

Thirdly, starting in the mid-1990s, more sophisticated machine learning algorithms (than just simple thresholding) were available, which all have extensively been explored and applied for the aforementioned polyp-detection experiments based on the extracted texture features, such as "Bayes classifiers" [33, 34], "linear discriminators" [29], "nearest neighbor classifier" [20, 28-30, 39, 40], "artificial neural networks" (ANNs) [22-24, 26-28, $32,36,41,42]$, "support vector machines" (SVMs) [10, 21, $28,30,31,39,40]$, and "random forest classifiers" [43].

Within the AI-age of statistical learning, the understanding and availability of these new powerful machine learning tools led to an explosive growth for the ability to detect meaningful signals within (colonoscopy image) data [5]. Furthermore, in this era the necessary computing power was at hand, allowing the extraction of the 

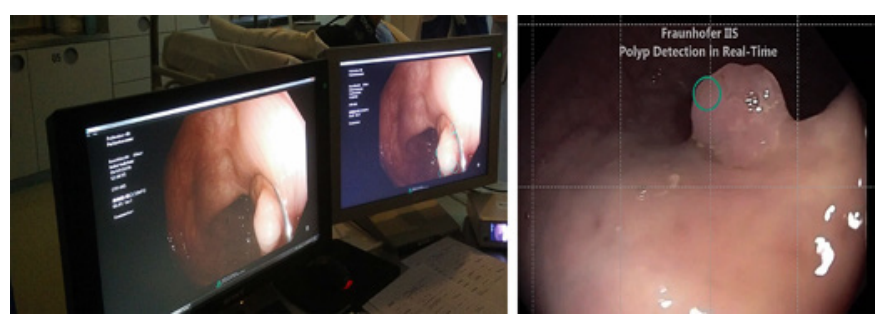

Fig. 6. The Fraunhofer IIS "KoloPol" polyp detection system (left) during the prospective clinical trial. The left monitor displays the original colonoscopy video stream, on the right monitor possible polyps are visually augmented using a green circle.

complex (color) texture features of the related experiments to be computed within in a manageable timeslot. Nevertheless, almost all described experiments were based on colonoscopic image data collections in the range between some hundreds and a maximum of approximately 2,500 static images $[39,40]$.

\section{First Clinical Trial}

Based on the abovementioned availability of textureand structure-based features for the image-based characterization and statistical classification of tissues in the colon, the highlight of this era (and also representing its virtual end) was denoted by the first published system for realtime, low-delay polyp detection based on HD video streams during a prospective screening colonoscopy study [44, 45] (Fig. 6). To our knowledge, in this study for the first time such a complete system for automated polyp detection was used during clinical routine colonoscopies, and yielded detection rates of approximately $75 \%$ of adenomas with respect to the endoscopist. The missed lesions were mainly related to small $(<6 \mathrm{~mm})$ and flat polyps. Nevertheless, as a machine or deep learning approach is always as good as the available training data, it can be assumed that with more and better image data collections usable for the development of polyp-detection schemes, their accuracy with respect to lesion detection will improve.

\section{Era of Featureless Modelling}

These conclusions directly led into the recent era (past 5 years) of "featureless modelling." Similar to the previous era, two main driving forces can be identified and observed.

\section{The Big Data Phenomenon}

The first force is related to the "Big Data" phenomenon. With social networks and online commerce having been booming in the past decade, the amount, usage, and value of any type of data has risen immensely. This increasing amount of data is not only related and limited to social media (such as images, videos, posts, messages, comments, and interactions), or from e-commerce (product placements and evaluations, purchases, transfers, rat- ings, interactions, customer profiles), but also includes any data from the "quantify yourself" movements (running and biking distances and speeds, geo-data, vital signs, behaviors, etc.) and any type of clinical data. With the perception that "data will be the oil or resources of the 21st century" (a phrase coined by Germany's chancellor Angela Merkel), many national and international, privateand public-funded activities were launched concerning the organized collection and provision of large-scale data for the development and evaluation of new approaches and algorithms for their analysis. Even though the idea of organized data collection and provision of medical data is quite old [46, 47], no real necessity was seen within the community to share valuable data. The essential push within the research field of polyp detection came in 2015 when, as part of the international conference of MICCAI ("Medical Image Computing and Computer-Assisted Intervention"), for the first time a "polyp-detection challenge" was hosted and the results were presented. Specifically, the success of this challenge was based on the availability of various annotated data collections, namely the ETIS Larib Polyp, the CVC-ColonDB, and the CVC clinical databases [48, 49], consisting of 194, 300, and 612 manually annotated colonoscopy images, respectively. Furthermore, the CVC-Clinic2017 [49] data collection consists of two sets of 18 colonoscopy videos each, and in total corporate 30,687 single frames, while the ASU-Mayo Clinic colonoscopy video database includes 38 colonoscopy videos with durations of between 4 and $61 \mathrm{~s}$ and a frame rate of $30 \mathrm{fps}$. [43]. The image data in these data collections have been obtained with endoscopy systems for various vendors and depict polyps and lesions of unknown classifications in many different orientations, sizes, angles, and views. Since then the Kvasir image collection has also been published $[50,51]$, originally comprising 8,000 images from 8 classes throughout the gastrointestinal tract and including 1,000 images with labelled polyps. Finally, the EDD2020 challenge [52] has recently provided a new training data set for multiclass disease detection, containing 386 endoscopic images labeled with 684 bounding boxes and 502 segmentation masks. Table 1 provides an overview of the currently publicly available image data collections with respect to the development, training, and evaluation of polyp-detection systems.

\section{Artificial Neural Networks and Deep Learning}

The second force were the enhancements in the field for ANNs, and specifically their extensions to deep neural networks, also known as "deep learning." The ANNs from the 1990s were mainly used as one type of classifier amongst others, where a set of features (e.g., the discussed texture features) where used as an input vector. This input vector was then transformed and transferred through the neural network architecture by a set of well-arranged 
Table 1. Overview of the currently publicly available image data collections used for the development, training, and evaluation of automated polyp-detection systems

\begin{tabular}{lllcc}
\hline & Origin & Images, $n$ & Sequences, $n$ & $\begin{array}{l}\text { Image size, } \\
\text { pixels }\end{array}$ \\
\hline CVC-CLINIC2015 & Spain & 612 & 31 & $388 \times 244$ \\
\hline CVC-ColonDB & Spain & 300 & 15 & \\
\hline Etis Larib & France & 196 & 34 & $1,225 \times 966$ \\
\hline ASU-Mayo Clinic Colonoscopy & USA & 36,570 & 38 & $712 \times 480$ \\
Video Data & & & 36 & $856 \times 480$ \\
\hline CVC-Clinic2017 & Spain & 30,687 & - & $720 \times 576$ \\
\hline Kvasir image collection & Norway & 8,000 & & $1,920 \times 1,072$ \\
\hline EDD2020 & & & - & - \\
\hline
\end{tabular}

weights, with at most three hidden layers, and finally provided a (binary) result in the output layer. As mentioned before, within the scope of colonoscopy, such ANNs were used to differentiate between benign tissue and possible lesions [21-28, 36, 41, 42]. With the rise of "deep neural networks," on one hand the amount of hidden layers between the input and output layers of an artificial networks was increased, and can currently consist of up to 100 layers or more, each one related to a set of weights to be automatically adjusted by an optimization scheme, known as "error-backpropagation." On the other hand, the original input vector denoting the aforementioned structure and texture features was replaced by a so-called set of convolutional layers, which are able to extract information (texture, structure, and color) directly from the images themselves, and thus make the manual definition and computation of such features unneeded. More specifically, the optimization of the weights related to the convolutional layers can even be directly included into the backpropagation scheme of the deep neural network. These deep convolutional networks (DCNNs) can be considered as featureless classification models. One drawback and simultaneous strength of such DCNNs is their need for large labelled image collections in order to train the network to all possible eventualities appearing in the images and without defining any textural descriptors. Besides the need of adequate and large-scale data to train such a DCNN, the underlaying net architecture plays a great role for the achieved results. These architectures vary in the amount and sizes of the convolutional and fully connected layers as well as the used activation and error functions.

Recent Developments Based on Deep Learning

For the automated detection in colonoscopic images and sequences, in the past 5 years several architectures of
DCNN have been proposed. The availability of the aforementioned public as well as private data collections were essential for these developments. For example, Billah et al. [25] made use of Wavelets for the characterization of image regions which were then classified by deep neural networks. For the training of the network more than 8,000 images from 50 colonoscopy sequences were used, and an additional 7,000 images were used for the evaluation. A detection rate of $F 1=0.98$ was reported for polyps, including adenoma, hyperplastic, and serrated polyps. Mohammed et al. [53] used the Mayo data collection for a Y-Net-architecture and achieved a mean detection rate of $0.85 \%$ on the abovementioned ASU Mayo clinic video data (Table 1). Misawa et al. [54] proposed a 3D-CNN and trained it with 63,135 images with and 133,496 pictures without polyps. For evaluation, 50 colonoscopy sequences with and 30 without polyps were used, yielding a sensitivity of 0.76 and a specificity of 0.90 . A Faster-RCNN architecture was used by both Mo et al. [49] as well as Shin et al. [55]. Mo et al. [49] trained their network using the abovementioned CVC-Clinic2017 data collection (Table 1) and tested it on 912 images with an accuracy of $0.89 \%$. Shin et al. [55] extend the CVC Clinic data collection with 28,600 synthetically generated images and tested it with a mean detection rate of $92 \%$ on the $19,400 \mathrm{im}-$ ages from the Mayo collection. Using an extension of the Faster-R-CNN, the Masked-R-CNN, Zobel et al. [56] reached a combined detection and segmentation rate of $93 \%$ on their data collection of 5,000 image patches [39] and $86 \%$ on the CVC data set (Fig. 7). Urban et al. [57] trained several classical DCNN networks with up to 20 layers based on 8,641 images from 2,000 patients and yielded a detection rate of 0.96 based on 76,607 evaluation images from 20 sequences. Wang et al. $[58,59]$ trained a SegNet architecture on 5,545 images (3,634 with polyps) 
Fig. 7. Examples for automatically detected and segmented polyps using AI based on deep neural networks. Upper row, original data; center row, automatically detected and segmented polyps (in red); lower row, expert annotation as ground truth (in green). From left to right: not detected (false-negative) polyp, flat polyp, two very small polyps (one of them not annotated), small polyp, sessile polyp (image from $\mathrm{Zo}$ bel et al. [56]).

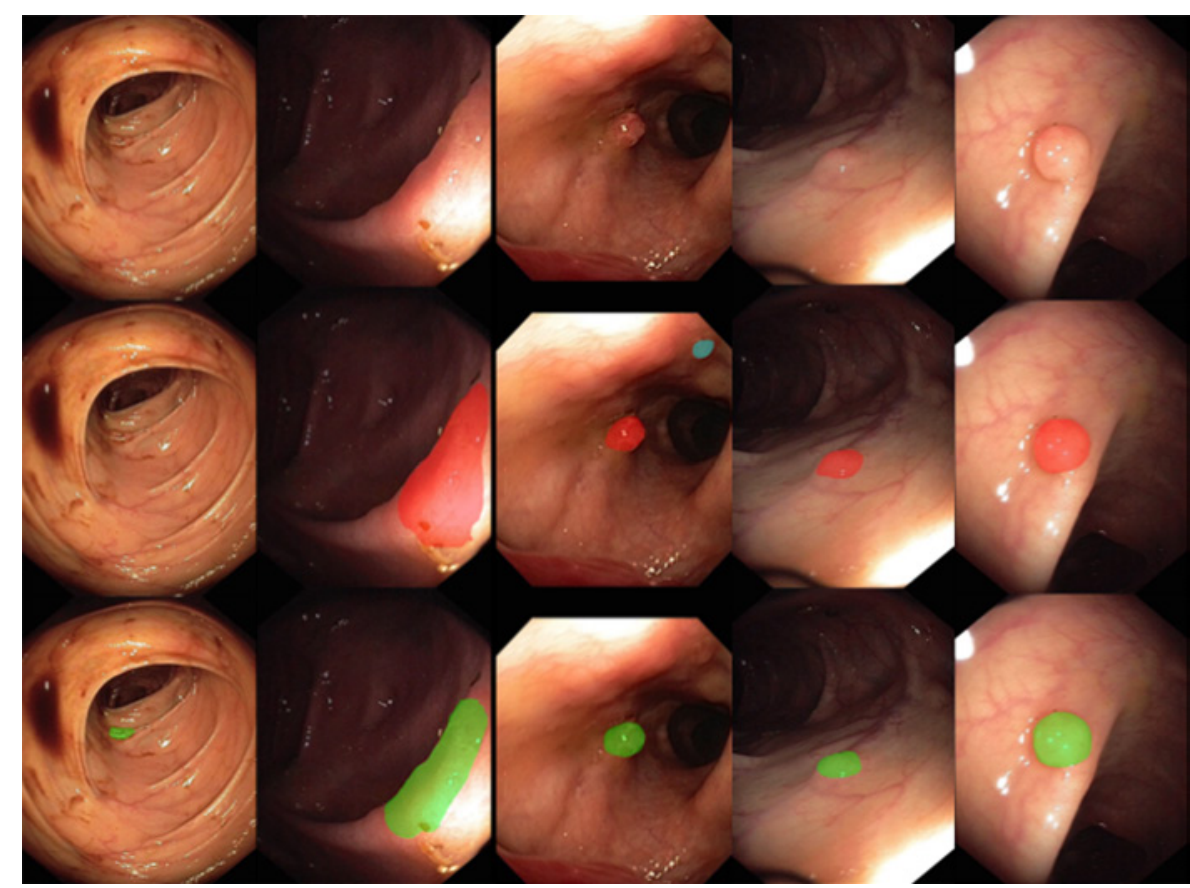

from 1,290 patients. The reported detection rate was $94 \%$ on 27,113 images (5,541 with polyps) from an additional 1,138 patients.

In summary, it can be seen that the achieved detection rates of $90 \%$ and above are tightly related to the training data used (Mo et al. [49] used 100,000 images, Misawa et al. [54] used nearly 200,000 images). Furthermore, the applied net architectures seem to play a crucial role combined with the mathematical approaches for the optimization and tuning of all the necessary parameters of a DCNN. Also, the evaluation of the diverse approaches is not made on the image or sequence level, but has already reached the patient level (Wang et al. [58, 59] with $>1,100$ patients, and Urban et al. [57] with 2,000 patients).

\section{Marked-Ready Systems Based on Deep Learning}

This leads to the observation that in the past year (November 2019 to July 2020) various commercially available products and systems have been brought to market, all of them using featureless DCNN architectures in order to achieve polyp detection directly (in real-time and/or post-processing) during colonoscopy and thus providing the endoscopist valuable hints to have a closer look. Namely, amongst these commercially available products are the GI Genius from Medtronics (USA) [60], CAD EYE from FujiFilm (Japan) [61], DISCOVERY by Pentax Medical (Japan) [62], AI4G from AI4GI Corp. (Canada) and Olympus America (USA) [63, 64], or EndoBrain from Cybernet Systems (Tokyo, Japan) [65].

While the first three systems address the idea of automated computer-assisted polyp detection (CADe), the AI4GI and EndoBrain technology address the next step, namely the characterization and differentiation of suspicious lesions in the colon in order to predict a polyp's pathology as either "neoplastic" or "non-neoplastic" based on in vivo microscopic imaging obtained by an endocytoscope (EndoBrain) [65] or NBI (AI4GI) image data [63, 64].

For the GI Genius system (Medronic), the first published results based on 685 subjects [60] propose that the adenoma detection rate was significantly higher using the CADe system (54.8\%) than in the control group (40.4\%), as the number of AI-based detected adenomas per colonoscopy was significantly higher than in the control group. In a small initial detection and differentiation study with approximately 2,000 images and 6 colonoscopists, the CAD EYE system (FujiFilm) was rated better (sensitivity $94.1 \%$, specificity $94.9 \%$ ) than beginners (sensitivity $96.4 \%$, specificity $79.7 \%$ ) and almost as good as experts (sensitivity 95.8\%, specificity 95.6\%) [61]. Such systems have recently been evaluated within a meta-study [66] with the conclusion that "the incorporation of artificial intelligence as an aid for the detection of colorectal neoplasia results in a significant increase of the detection of colorectal neoplasia."

For the AI4GI system it was reported that the AI model did not generate sufficient confidence to predict the histology of 19 polyps (15\%) from 135 NBI video sequences in the test set. For the remaining 106 diminutive polyps, a sensitivity of $98 \%$ and a specificity of $83 \%$ for the system was reported [64]. For the automated analysis, the EndoBrain system was initially trained based on 69,142 endocytoscopic images. It was reported that the system identified neoplasms with $96.9 \%$ sensitivity, $100 \%$ speci- 
ficity, and 98\% accuracy. In an application to narrowband images, the system differentiated "neoplastic" from "non-neoplastic" lesions with 96.9\% sensitivity, 94.3\% specificity, and 96.0\% accuracy (95\% CI 95.1-96.8). It was also reported that all values were significantly better than the reference values from trainees, and comparable to the experts [65]. However, endocytoscopic colonoscopic assessment is largely restricted to Asian investigators and specialized clinical research activities. Thus, whether this development will become routine for screening colonoscopy worldwide is uncertain. Other developments, like Endocuff, Endorings, cap-assisted colonoscopy, and FUSE, have shown to improve colonoscopic screening [67]. However, in combination with improved automated detection systems and AI a near 100\% detection rate for any type of colorectal polyps appears to be reachable, provided that in a clean colon a careful colonoscopic technique is used with inspection or depiction of all front and back sides of the folds, including views to hidden spaces of the colon. If this technical prerequisite can be afforded by the experienced endoscopist, automated polyp detection, which needs only seconds to recognize pathological patterns, can substantially improve the diagnostic efficacy of colonoscopy to nearly $100 \%$. In difficult regions, CADe time is probably superior to the endoscopist's eye, as the endoscopist may sometimes need a short time to overview and scan the region, for example in angled regions, or when the colonoscope is sliding back.

Thus, in coherence with Vorhies [5], this second age of AI extends back many decades and has brought forward three major examples of new technologies, namely machine learning, big data, and featureless modeling.

\section{Discussion: Age of Contextual Adaptation}

Even though the first systems for AI-supported detection of polyps have entered the market and are currently part of clinical trials, the underlaying mathematical approaches of DCNNs still reflect the idea of so-called weak AI. This term relates to the idea that such systems are designed to solve exactly one problem, namely the detection of polyps. As can be seen, the available systems and approaches solve this task surprisingly well, and possibly better than a novice endoscopist. Nevertheless, that is all these systems can do. In contrast to a human endoscopist, these systems and approaches cannot adapt easily to new inputs or data. Hence, if the pre-assumptions made for the design of such systems change (such as the use of white light illumination, the spatial resolution of the image sensors, or the visible optical field of the sensor), these systems have to be retrained with a plethora of data, while a human can easily adapt to new visual settings of endoscopy.
Similarly, as a human endoscopist always uses holistic (anamnestic, demographic, laboratory, medication) information about the patient, and thus relates any finding and the final diagnosis to this knowledge, currently available AI systems are built to solve only one single and narrow task, namely the detection of any polypoid, suspicious or - at best - neoplastic lesion, then differentiation of the lesion to non-neoplastic or neoplastic etiology. Thus, these systems are also referred to as "weak AI" systems. Nevertheless, this observation leaves room for some future visions and possible ideas for the development of AI within colonoscopy.

First of all, AI systems will need to be automatically aware of and have to adapt to their context. Thus, such a system automatically has to detect and differentiate if the colonoscope is inserted, is withdrawn, or a polypectomy is currently being executed. Currently, an automated polyp detection only in the second case (withdrawal) is feasible. However, in an extended future version, it is easily thinkable that an AI-based support system automatically tracks and knows (and displays) in which section of the colon the tip of the system is currently located (sigmoid, ascending, descending, transverse, descending, cecum), how long the visual assessment of each section has taken, how often polypoid or suspicious lesions have been recognized, what type of lesions or polyps have been detected (e.g., automatic AI-based differentiation [52, 68] and staging). In this regard it is important for the endoscopist to know in real-time where and how many lesions are present, and (by relating the detected information to the patient's data) AI can help to make automatic suggestions about the diagnosis, medication, treatment, and predictions about the outcome. Nevertheless, as a prerequisite for such an AI-based support, a sufficient amount of clinical data and outcomes will have to be collected and used to train such enhanced AI systems. Furthermore, by adding explanation components to such systems, they will advance from AI systems to systems with explainable AI (XAI).

\section{Conclusion}

We have provided a brief description of the three ages of AI-based colonoscopy detection, from early experiments based on handcrafted geometric features and simple decision schemes, via texture-based approaches using machine learning methods for tissue classification, up to current developments using featureless methods based on convolutional deep neural networks for automated polyp detection. We have briefly described the necessity of well-annotated data for the research and development of such new methods, starting with some dozen digitized images in the early days and currently using some thou- 
sand complete colonoscopy sequences for the evaluation of marked-ready systems. Finally, some possible future developments regarding the next stages and steps of AIbased colonoscopy have been envisioned.

\section{Conflict of Interest Statement}

The authors have no conflicts of interest to declare.

\section{Funding Sources}

No funding bodies were associated with this research.

\section{Author Contributions}

T.W. conceived of and wrote the manuscript. M.R. contributed to the medical part of the manuscript.

\section{References}

1 Shen L, Margolies LR, Rothstein JH, Fluder E, McBride R, Sieh W. Deep learning to improve breast cancer detection on screening mammography. Sci Rep. 2019 Aug;9(1):12495.

2 Yala A, Lehman C, Schuster T, Portnoi T, Barzilay R. A deep learning mammographybased model for improved breast cancer risk prediction. Radiology. 2019;292:60-66.

3 Abdelhafiz D, Yang C, Ammar R, Nabavi S. Deep convolutional neural networks for mammography: advances, challenges and applications. BMC Bioinformatics. 2019; 20(Suppl 11):281.

4 Heath M, Bowyer K, Kopans D, Moore R, Kegelmeyer W. The Digital Database for Screening Mammography. 5th International Workshop on Digital Mammography. Toronto, June 2000.

5 Vorhies W. The Three Ages of AI - Figuring Out Where We Are. Data Science Central [2017 May 9]. Available from: https://www. datasciencecentral.com/profiles/blogs/thethree-ages-of-ai-figuring-out-where-we-are.

6 Chettaoui H, Thomann G, Ben Amar C, Redarce T. Extracting and tracking colon's pattern from colonoscopic images. 3rd Canadian Conference on Computer and Robot Vision (CRV'06), Quebec, 2006.

7 Tjoa MP, Krishnan SM, Kugean C, Wang P, Doraiswami R. Segmentation of clinical endoscopic image based homogeneity and hue. Annual International Conference of the IEEE Engineering in Medicine and Biology Society, Istanbul, 2001.

8 Tjoa MP, Krishnan SM, Doraiswami R. Automated diagnosis for segmentation of colonoscopic images using chromatic features, IEEE CCECE2002. Canadian Conference on Electrical and Computer Engineering. Winnipeg, 2002. https://doi.org/10.1109/CCECE.2002. 1013115.

9 Dhandra BV, Hegadi R, Hangarge M, Malemath VS. Analysis of abnormality in endoscopic images using combined HSI color space and watershed segmentation. 18th International Conference on Pattern Recognition (ICPR'06), Hong Kong, 2006. https://doi. org/10.1109/ICPR.2006.268.

10 Iakovidis DK, Maroulis DE, Karkanis SA, Brokos A. A comparative study of texture features for the discrimination of gastric polyps in endoscopic video. 18th IEEE Symposium on Computer-Based Medical Systems (CBMS'05), Dublin, 2005. https://doi.org/ 10.1109/CBMS.2005.6.
11 Tjoa MP, Krishnan SM, Zheng MM. A novel endoscopic image analysis approach using deformable region model to aid in clinical diagnosis. 25th Annual International Conference of the IEEE EMBS, Cancun, September 17-21, 2003. https://doi.org/10.1109/ IEMBS.2003.1279862.

12 Hiremath PS, Dhandra BV, Hegadi R, Rajput GG. Abnormality detection in endoscopic images using color segmentation and curvature computation. In: Pal NR, Kasabov N, Mudi RK, Pal S, Parui SK, editors. Neural Information Processing. ICONIP. Lecture Notes in Computer Science. Volume 3316. Berlin, Heidelberg: Springer; 2004.

13 Krishnan SM, Yang X, Chan KL, Kumar S, Goh PM. Intestinal abnormality detection from endoscopic images. Proc IEEE EMB. 1998; 2:895-8

14 Phee SJ, Ng WS, Chen IM, Seow-Choen F, Davies BL. Interpreting images with a computer to automomatically maneuver the colonoscope. IEEE Eng Med Biol Mag. 1998; 17(3):81-8.

15 Hwang S, Oh J, Tavanapong W, Wong J, deGroen P. Polyp detection in colonoscopy video using elliptical shape feature. Proc IEEE ICIP. 2007;(2):465-8.

16 Ameling S, Wirth S, Shevchenko N, Wittenberg T, Paulus D, Münzenmayer C. Detection of lesions in colonoscopic images: a review. World Congress on Medical Physics and Biomedical Engineering, Munich, 2009. https:// doi.org/10.1007/978-3-642-03882-2_265.

17 Kudo S, Tamura S, Nakajima T, Yamano H, Kusaka H, Watanabe H. Diagnosis of colorectal tumorous lesions by magnifying endoscopy. Gastrointest Endosc. 1996 Jul;44(1):8-14.

18 Participants in the Paris Workshop. The Paris endoscopic classification of superficial neoplastic lesions: esophagus, stomach, and colon: November 30 to December 1, 2002. Gastrointest Endosc. 2003 Dec;58(6Suppl):S3-43.

19 Humeau-Heurtier A. Texture feature extraction methods: a survey. IEEE Access. 2019;7: 8975-9032.

20 Shevchenko N, Mühldorfer S, Wittenberg T, Münzenmayer M. Untersuchung von Texturanalysemethoden zur automatischen Polypenerkennung. CURAC, Leipzig, 2008.

21 Münzenmayer C, Shevchenko N, Mühldorfer S, Wittenberg T. Unser's statistical features versus color and position for automated polyps recognition. Int J CARS. 2009;2(S1):S368.
22 Karkanis S, Magoulas GD, Grigoriadou M, Schurr MO. Detecting abnormalities in colonoscopic images by textural description and neural networks. Advanced Course in Artificial Intellegence - Workshop on Machine Learning in Medical Applications, Chania, 1999.

23 Karkanis S, Magoulas GD, Theofanous N. Image recognition and neuronal networks: intelligent systems for the improvement of imaging information. Minim Invasive Ther Allied Technol. 2000;9(3-4):225-30.

24 Plagianakos VP, Magoulas GD, Vrahatis MN. Tumor detection in colonoscopic images using hybrid methods for on-line neural network training. 4th International Conference "Neural Networks and Expert Systems in Medicine and Healthcare", Milos Island, June 20-22, 2001.

25 Billah M, Waheed S, Rahman MM. An automatic gastrointestinal polyp detection system in video endoscopy using fusion of color wavelet and convolutional neural network features. Int J Biomed Imaging. 2017;2017: 9545920. https://doi.org/https://doi.org/ $10.1155 / 2017 / 9545920$.

26 Tjoa MP, Krishnan SM. Feature extraction for the analysis of colon status from the endoscopic images. Biomed Eng Online 2003;2:9.

27 Magoulas GD, Plagianakos VP, Vrahatis MN. Neural network-based colonoscopic diagnosis using on-line learning and differential evolution. Appl Soft Comput. 2004;4(4):369-79.

28 Hafner M, Gangl A, Wrba F, Thonhauser K, Schmidt HP, Kastinger C, et al. Comparison of k-NN, SVM, and NN in Pit Pattern classification of zoom-endoscopic colon images using co-occurrence histograms. 5th International Symposium on Image and Signal Processing and Analysis, Istanbul, 2007.

29 Esgiar AN, Sharif BS, Naguib RN, Bennett MK, Murray A. Texture descriptions and calssification for pathological analysis of canercous colonoscopic muosa. IEEE 7th International Conference on Image Processing and Its Applications, Manchester, 1999.

30 Manivannan S, Wang R, Trucco E. Extended Gaussian-filtered local binary patterns for colonoscopy image classification. 2013 IEEE International Conference on Computer Vision Workshops, Sydney, 2013.

31 Alexandre LA, Nobre N, Casteleiro J. Color and position versus texture features for endoscopic polyp detection. IEEE International Conference BioMedical Engineering and Informatics, 2008. 
32 Wang P, Krishnan SM, Kugean C, Tjoa MP. Classification of endoscopic images based on texture and neural network. 23rd EMBS Conference, Istanbul, 2001.

33 Häfner M, Brunauer L, Payer H, Resch R, Wrba F, Gangl A, et al. Pit Pattern classification of zoom-endoscopical colon images using DCT and FFT, 20th IEEE Int. Symp. on ComputerBased Medical Systems 2007. pp. 159-64.

34 Hafner M, Gangl A, Brunauer L, Payer H, Resch R, Uhl A, et al. Pit pattern classification of zoom-endoscopical colon images using evolved Fourier feature vectors. IEEE Workshop on Machine Learning for Signal Processing, Thessaloniki, 2007. pp. 99-104

35 Stehle S, Auer R, Gross S, Behrens A, Wulff J, Aach A, et al. Classification of colon polyps in NBI endoscopy using vascularization feature. Med Imaging. 2009;72602S:72602S.

36 Karkanis SA, Iakovidis DK, Maroulis DE, Magoulas GD, Theofanous NG. Tumor recognition in endoscopic video images using artificial neural network architectures. 26th Euromicro Conference. Informatics: Inventing the Future. Maastricht, 2000. pp. 423-9. h t t p s:// d oi.org/10.1109/E U R MIC.2000.874524.

37 Häfner M, Kwitt R, Uhl A, Wrba F, Gangl A, Vécsei A. Computer-assisted pit-pattern classification in different wavelet domains for supporting dignity assessment of colonic polyps. Pattern Recogn. 2009;42(6):1180-91.

38 Park SY, Sargent D, Spofford I, Vosburgh KG, A-Rahim Y. A colon video analysis framework for polyp detection. IEEE Trans Biomed Eng. 2012 May;59(5):1408-18.

39 Nowack S, Benz M, Münzenmayer C, Raithel M, Prinzen M, Mühldorfer S, et al. Unterscheidung von Polypen und Hintergrundgewebe in statischen Koloskopieaufnahmen. CURAC 2015, Bremen. pp. 201-4. https://curac.org/images/advportfoliopro/images/CURAC2015/Programm-CURAC2015.pdf

40 Wittenberg T, Nowack S, Prinzen M, Krappe S, Raithel M, Mühldorfer S, et al. Differentiation of neoplastic lesions and healthy tissue in HD colonoscopy images based on color texture features. Presented at SPIE Medical Imaging: Computer-Aided Diagnosis, San Diego, March 2016.

41 Karkanis S, Magoulas GD, Grigoriadou M, Schurr MO. Detecting abnormalities in colonoscopic images by textural description and neural networks. In: Proceedings of Workshop on Machine Learning in Medical Applications. 1999. p. 59-62.

42 Karkanis SA, Maroulis DE, Iakovidis DK, Maroulis DE, Schurr MO. On the importance of feature descriptors for the characterisation of texture. 4th World Multiconference on Systems, Cybernetics and Informatics (SCI 2000). Volume VI. Orlando (Florida): Image Acoustic, Speech and Signal Processing, Part II; 2000. pp. 96-101.

43 Tajbakhsh N, Gurudu SR, Liang J. Automated polyp detection in colonoscopy videos using shape and context information. IEEE Trans Med Imaging. 2016;35(2):630-43.

44 Klare P, Sander C, Prinzen M, Münzenmayer C, Nowack S, von Delius S, Wittenberg T. Computer assisted detection of polyps during colonoscopy - results from an initial technical study. Biomed Eng Biomed Tech. 2017; 62(S1): 15

45 Klare P, Sander C, Prinzen M, Haller B, Nowack S, Abdelhafez M, et al. Automated polyp detection in the colorectum: a prospective study (with videos). Gastrointest Endosc. 2019 Mar;89(3):576-582.e1.

46 Wittenberg T, Horsch A. Current state of the reference image database concept of EFMI WG MIP. In: Blobel B, Gell G, Hildebrand C, Engelbrecht R (eds.): Proceedings of the EFMI Special Topics Conference. Contribution of medical informatics to health-integrated clinical data and knowledge to support primary, secondary, tertiary home care. Munich, May 2004. pp. 129-30.

47 Horsch A, Prinz M, Schneider S, Sipilä O, Spinnler K, Valee JP, et al. Establishing an international reference image database for research and development in medical image processing. In: Wittenberg $\mathrm{T}$, Hastreiter $\mathrm{P}$, Hoppe U, Handels H, Horsch A, Meinzer HP, editors. Bildverarbeitung für die Medizin 2003. Informatik aktuell. Berlin: Springer; 2003. p. 263-7. https://link.springer.com/ chapter/10.1007/978-3-642-18993-7_74

48 Bernal J, Tajkbaksh N, Sanchez FJ, Matuszewski BJ, Hao Chen, Lequan Yu, et al. Comparative validation of polyp detection methods in video colonoscopy: results from the MICCAI 2015 endoscopic vision challenge. IEEE Trans Med Imaging. 2017 Jun;36(6):123149.

49 Mo X, Kao T, Wang Q, Wang G. An efficient approach for polyps detection in endoscopic videos based on Faster R-CNN. arXiv. 2018; 1809:01263.

50 Pogorelov K, Randel K, Griwodz C, Sigrun E, Lange $\mathrm{T}$, Johansen $\mathrm{D}$, et al. A multi-class image dataset for computer aided gastrointestinal disease detection. Proceedings of the Multimedia Systems Conference (MMSys). Taiwan: ACM; 2017. pp. 164-169.

51 Debesh J, Smedsrud PH, Riegler MA, Halvorsen $\mathrm{P}$, de Lange T, Johansen D, et al. KvasirSEG: a segmented polyp dataset. arXiv. 2020; 1911:07069v1.

52 Ali S, Ghatwary N, Braden B, Lamarque D, Bailey A, Realdon S, et al. Endoscopy disease detection challenge. arXiv. 2020;2003:03376.

53 Mohammed A, Yilderim S, Farup I, Perdersen M, Havde O. Y-Net: A deep convolutional neural network for polyp detection. 2018 arXiv. 2018;1806:01907v1

54 Misawa M, Kudo SE, Mori Y, Cho T, Kataoka $\mathrm{S}$, Yamauchi A, et al. Artificial intelligenceassisted polyp detection for colonoscopy: initial experience. Gastroenterology. 2018 Jun; 154(8):2027-2029.e3.

55 Shin Y, Ali Quadir, H, Aabakken L, Bergsland J, Balasingham I. Automatic colon polyp detection using region based deep CNN and post learning approaches. IEEE Access. 2018; 6: 40950-62. https://doi.org/10.1109/ACCESS.2018.2856402.

56 Zobel P, Rathke M, Mühldorfer S, Wittenberg T. Computer aided detection of polyps in white-light-colonoscopy images using deep neural networks. Curr Dir Biomed Eng. 2019; 5(1):231-4.
57 Urban G, TriPathi P, Alkayali, T, Mittal M, Jalali F, Kames W, et al. Deep learning localizes and identifies polyps in real time with 96\% accuracy in screening colonoscopy. Gastroenterology. 2019;155:1069-78.

58 Wang P, Xiao X, Glissen Brown JR, Berzin TM, Tu M, Xiong F, et al. Development and validation of a deep-learning algorithm for the detection of polyps during colonoscopy. Nat Biomed Eng. 2018 Oct;2(10):741-8.

59 Wang P, Bezin TM, Brown JR, Bharadwaj S, Becq A, Xiao X, et al. Real-time automatic detection system increases colonoscopic polyp and adenoma detection rates: a prospective randomised controlled study. Gut. 2019;68: 1813-9. https://doi.org/10.1136/gutjnl-2018317500.

60 Repici A, Badalamenti M, Maselli R, Correale L, Radaelli F, Rondonotti E, et al. Efficacy of Real-Time Computer-Aided Detection of Colorectal Neoplasia in a Randomized Trial. Gastroenterology. 2020 Aug;159(2):512-520. e7.

61 Weigt J, Neumann H, Repici A, Hassan C. Mit Hilfe eines validierten Polypendetektions- und Charakterisierungssystems können unerfahrene Untersucher Expertennieveau erreichen. Z Gastroenterol. 2020; 58(08):e181.

62 Pentax Medical. HOYA Group PENTAX Medical cleared CE Mark for DISCOVERYTM, an AI assisted polyp detector [2019 Dec 16]. Available from: https://www.pentaxmedical. com/pentax/en/92/1/HOYA-Group-PENTAX-Medical-Cleared-CE-Mark-for-DISCOVERY-an-AI-Assisted-Polyp-Detector.

63 Chahal D, Byrne MF. A primer on artificial intelligence and its application to endoscopy. Gastrointest Endosc. 2020 Oct;92(4):813820.e4.

64 Byrne MF, Chapados N, Soudan F, Oertel C, Linares Pérez M, Kelly R, et al. Real-time differentiation of adenomatous and hyperplastic diminutive colorectal polyps during analysis of unaltered videos of standard colonoscopy using a deep learning model. Gut. 2019 Jan; 68(1):94-100.

65 Kudo SE, Misawa M, Mori Y, Hotta K, Ohtsuka $\mathrm{K}$, Ikematsu $\mathrm{H}$, et al. Artificial Intelligence-assisted System Improves Endoscopic Identification of Colorectal Neoplasms. Clin Gastroenterol Hepatol. 2020 Jul;18(8):18741881.e2.

66 Hassan C, Spadaccini M, Iannone A, Maselli R, Jovani M, Chandrasekar VT, et al. Performance of artificial intelligence for colonoscopy regarding adenoma and polyp detection: a meta-analysis. Gastroint Endoscop. 2020. doi: 10.1016/j.gie.2020.06.059.

67 Rex DK, Repici A, Gross SA, Hassan C, Ponugoti PL, Garcia JR, et al. High-definition colonoscopy versus Endocuff versus EndoRings versus full-spectrum endoscopy for adenoma detection at colonoscopy: a multicenter randomized trial. Gastrointest Endosc. 2018 Aug; 88(2):335-344.e2

68 Yang YJ, Cho BJ, Lee MJ, Kim JH, Lim H, Bang CS, et al. Automated classification of colorectal neoplasms in white-light colonoscopy images via deep learning. J Clin Med. 2020 May;9(5):1593. 